\title{
CLASSIFICAÇÃO DO GRAU DE COMPROMETIMENTO MOTOR E DO ÍNDICE DE MASSA CORPÓREA EM CRIANÇAS COM PARALISIA CEREBRAL
}

\section{CLASSIFICATION OF GROSS MOTOR FUNCTION INJURY AND BODY MASS ÍNDEX IN CHILDREN WITH CEREBRAL PALSY}

\author{
Karina Emi Shigekawa de Souza ${ }^{1}$ \\ Andréia Naomi Sankako ${ }^{2}$ \\ Sebastião Marcos Ribeiro de Carvalho ${ }^{3}$ \\ Lígia Maria Presumido Braccialli ${ }^{4}$
}

Souza KES et al. Classificação do grau de comprometimento motor e do índice de massa corpórea em crianças com paralisia cerebral. Rev. Bras. Cresc. e Desenv. Hum. 2011; 21(1): $11-20$

\section{Resumo:}

Crianças com paralisia cerebral devido às desordens de movimento e postura podem ter o seu estado nutricional comprometido. Assim, este estudo tem como objetivo classificar o índice de massa corpórea de crianças com paralisia cerebral e verificar se existe relação com o grau de comprometimento da função motora grossa. Para tanto, participaram do estudo 20 crianças com paralisia cerebral, com idade entre dois e 14 anos, do sexo masculino e feminino, atendidas em um centro de reabilitação com acompanhamento de fisioterapia, terapia ocupacional e fonoaudiologia. Todas foram classificadas de acordo com a escala de classificação da função motora grossa. Foram realizadas as medidas antropométricas de cada criança: peso; comprimento calcanhar-joelho; estatura estimada e índice de massa corpórea. O índice de massa corpórea foi classificado de acordo com o percentil do índice de massa corpórea de crianças e adolescentes do Center for Disease Control and Prevention. Os dados obtidos não permitiram identificar relação entre a classificação da função motora grossa e índice de massa corpórea. Talvez o número reduzido de participantes e o fato de todas as crianças serem acompanhadas em ambulatório de disfagia e, quando necessário em ambulatório de nutrição, possam ter influenciado esses resultados.

Palavras-chave: criança; paralisia cerebral; índice de massa corporal.

Faculdade de Medicina de Marília- FAMEMA.

2 Faculdade de Filosofia e Ciências, Unesp - Universidade Estadual Paulista, Marília.

3 Faculdade de Filosofia e Ciências, Unesp - Universidade Estadual Paulista, Marília.

4 Faculdade de Filosofia e Ciências, Unesp - Universidade Estadual Paulista, Marília.

Correspondência para: Dra. Lígia Maria Presumido Braccialli, Departamento de Educação Especial, Faculdade de Filosofia e Ciências, Unesp - Universidade Estadual Paulista, Avenida Hygino Muzzi Filho, 737, Caixa Postal: 181, C.E.P.: 17.525-900, Marília, São Paulo, Telefone: 5514 3402-1331, Fax: 5514 3402-1331. E-mail: bracci@marilia.unesp.br 


\begin{abstract}
:
Children with cerebral palsy due to movement and posture disorders might have an injured nutritional state. This study's objective was to classify the body mass index of children with cerebral palsy and verify its relation with the injury level of gross motor function. Twenty children, male and female sex, with cerebral palsy with ages between 2 and 14 years participated in the study. They were chosen from a rehabilitation center with physiotherapy, occupational and speech therapy accompaniment. Children were classified according to the gross motor function classification scale. The anthropometric measures of each child weight, heel-knee length, estimated stature and body mass index were gauged. The body mass index was classified according to the Center for Disease Control and Prevention's body mass index percentile calculator for children and teenagers. The obtained data didn't show any relation between the gross motor function and body mass index. The reduced number of participants and the fact of all children be accompanied in dysphagia clinics and, when necessary, nutrition clinics, might have influenced these results.
\end{abstract}

Key words: child; cerebral palsy; body mass index.

\section{INTRODUÇÃO}

A paralisia cerebral (PC) pode acometer os indivíduos de diferentes maneiras, por meio de alterações neuromusculares, com alterações de tônus, com a persistência de reflexos primitivos, rigidez e espasticidade. Tais alterações geram padrões de movimentos anormais que podem comprometer o desempenho funcional dessas crianças. A PC pode interferir na interação das crianças em contextos relevantes, por exemplo na aquisição e desempenho de marcos motores básicos como rolar, sentar, andar e também em atividades de vida diária, como tomar banho, vestir-se, locomover-se e alimentar-se ${ }^{1}$.

Devido às lesões que ocorrem no sistema nervoso central, algumas alterações motoras geradas pela PC tornam o movimento voluntário descoordenado, estereotipado e limitado $^{2}$. Consequentemente, a criança com PC pode apresentar, também, dificuldade na coordenação da musculatura oral, o que ocasiona uma dificuldade na deglutição ${ }^{3}$.

Estudos sobre problemas relacionados à alimentação de crianças com PC mostraram que a gravidade do tipo de PC está diretamente re- lacionada à dificuldade de deglutição, ao aumento de sintomas de disfagia e também a maiores complicações do estado nutricional ${ }^{4}$. Tais prejuízos na alimentação podem levar à perda de peso, que é uma característica marcante da PC e traduz a desnutrição progressiva frequentemente vista nessa condição.

A desnutrição na PC tem várias causas, sendo especialmente devida ao estado de hipermetabolismo resultante da inflamação pancreática e da ingestão alimentar diminuída por causa de dor. A sitofobia, medo de alimentar-se, é observada também em pacientes com alterações avançadas ${ }^{5}$.

Estudos realizados ao longo dos anos têm demonstrado elevada frequência de desnutrição em crianças com PC devido às dificuldades para se alimentar ${ }^{6,7}$. O acompanhamento do estado nutricional é um importante indicador a ser considerado no desenvolvimento de toda criança e, mais especificamente, naquelas com alterações neurológicas.

Para avaliar o estado nutricional de crianças e adolescentes desnutridos ou com risco de desnutrição e propor uma orientação adequada e individualizada, é realizada uma avaliação clínica dos componentes nutricionais. 
A mais comumente utilizada é o cálculo do índice de massa corpórea (IMC) segundo classificação da Organização Mundial da Saude $(\mathrm{OMS})^{8}$.

O cálculo do IMC utiliza os valores de peso e a estatura do indivíduo. O peso refere-se à soma de todos os componentes da composição corpórea, trata-se de uma medida aproximada das reservas totais da energia do corpo, e sua mudança reflete no equilíbrio de energia e proteína, além de traduzir casos de risco nutricional.

Porém, em crianças com paralisia cerebral, o acompanhamento do IMC torna-se difícil, principalmente porque algumas crianças são incapazes de se manter em pé, apresentam contraturas, escolioses, espasmos musculares involuntários e pouca cooperação devido à deficiência cognitiva.

Por isso, Stevenson ${ }^{9}$ desenvolveu fórmulas para estimar a estatura a partir de medidas segmentares e concluiu que, na impossibilidade de se medir a estatura diretamente, qualquer medida segmentar seria interessante para estimar a altura, porém a mais indicada seria o comprimento do joelho ao calcanhar.

A validação do método antropométrico descrito por esse autor permite um melhor acompanhamento nutricional da criança com $\mathrm{PC}^{7}$.

Em decorrência dos comprometimentos motores gerados pela paralisia cerebral e do quadro nutricional deficitário nesses individuos, para um bom acompanhamento clínico dessa população é essencial, além de calcular o IMC, classificar também o grau de função motora.

O sistema de classificação pela função motora grossa (GMFCS) foi desenvolvido por Palisano e colaboradores ${ }^{8,10}$ com o intuito de facilitar e uniformizar a avaliação do grau de acometimento motor em crianças com PC, e conquistou ampla aceitação para esse fim.

A GMFCS consta de cinco níveis de acometimento, tendo como base o controle de mo- vimentos voluntários que enfatizam o sentar e o andar. É realizada de acordo com a idade em quatro grupos: menores de dois anos, entre dois e quatro anos, entre quatro e seis anos e entre seis e doze anos ${ }^{2,8}$. Em 2007, a GMFCS foi expandida, incluiu a faixa etária de jovens de 12 a 18 anos de idade e enfatiza os conceitos presentes na Classificação Internacional de Funcionalidade, Incapacidade e Saúde (CIF) da Organização Mundial de Saúde ${ }^{11}$.

Assim, o objetivo é classificar o grau de comprometimento motor e o IMC de crianças com paralisia cerebral e analisar se existe relação entre esses dois dados.

\section{MÉTODO}

\section{Participantes}

Participaram do estudo 20 crianças com diagnóstico de paralisia cerebral, com idade entre dois e 14 anos, do sexo masculino e feminino, atendidas em um centro de reabilitação com acompanhamento de fisioterapia, terapia ocupacional e fonoaudiologia (Quadro 1). Além disso, as crianças que apresentavam disfagia eram atendidas também no ambulatório de disfagia disponível nesse mesmo centro de reabilitação.

Todos os pais ou responsáveis pelos participantes foram devidamente informados a respeito da pesquisa, concordaram e assinaram o Termo de Consentimento Livre e Esclarecido. O projeto foi submetido ao Comitê de ética em pesquisa da Faculdade de Filosofia e Ciências e foi aprovado com o parecer de número 685/2009.

\section{Procedimento de coleta de dados}

Primeiramente os participantes foram classificados de acordo com a escala GMFCS por meio de observação realizada pela pesquisadora responsável. Em seguida, foram realizadas as medidas antropométricas de cada participante: peso, comprimento do joelho ao cal- 
canhar, estatura estimada e cálculo do IMC. Foram utilizadas a Escala de classificação da função motora grossa - GMFCS, balança digital da marca Welmy RI W200 e paquímetro da marca CESCORF.

Para calcular o peso, a criança subiu na balança com roupas leves e sem sapatos, com os braços estendidos ao longo do corpo e com o olhar fixo à frente, nos casos em que essa condição não foi possível, o responsável subiu na balança, uma vez com a criança no colo, e depois, sem a criança. Dessa forma, calculou-se o peso do participante pela diferença entre o peso de ambos e o do acompanhante sozinho.

O comprimento do joelho ao calcanhar foi mensurado por meio de um paquímetro. Para tanto, cada participante foi colocado na posição sentada com a perna em flexão de 90 graus.

Para calcular a estatura estimada foi utilizada a seguinte fórmula: $\mathrm{E}=(2,69 \times \mathrm{CJ})+$ 24,2, sendo $\mathrm{E}=\mathrm{a}$ altura estimada , e CJ = comprimento do joelho ao calcanhar ${ }^{5,7,12}$.

O IMC foi calculado a partir da fórmula: $\mathrm{IMC}=\mathrm{P} / \mathrm{E}^{2}$, sendo $\mathrm{P}=$ peso $^{5}$. A classificação do IMC dos participantes foi realizada de acordo com a calculadora de percentil do IMC de crianças e adolescentes do Center for Disease Control and Prevention - CDC (Tabela 1). Essa calculadora fornece o IMC e o correspondente percentil do IMC para a idade e sexo no gráfico de crescimento da CDC. Ela é usada para crianças e adolescentes, com idade entre 2 e 19 anos com intuito de classificar o IMC em: Baixo Peso, Peso ideal, Sobrepeso e Obesidade ${ }^{8}$.

\section{Análise estatística}

A análise dos dados para as variáveis numéricas foi realizada por meio de número de indivíduos, média, desvio-padrão (DP), valor mínimo e valor máximo. A comparação entre os indivíduos, segundo o sexo, foi realizada por meio do teste t de Student para grupos independentes, segundo as variáveis numéricas: idade, peso, estatura e IMC. A verificação da normalidade dos dados foi realizada por meio do teste de Kolmogorov ${ }^{13}$. Adotou-se o nível de significância de 5\% de probabilidade para a rejeição da hipótese de nulidade.

\section{RESULTADOS}

O quadro 2 apresenta as caracteristicas dos participantes segundo IMC, percentil, classificação do IMC e GMFCS.

Na Tabela 2, são demonstradas as características gerais dos participantes do estudo, segundo a classificação do IMC, conforme o CDC. Observa-se um maior número de crianças classificadas com peso ideal, com o IMC em média de $16,2 \mathrm{Kg} / \mathrm{m}^{2}$, enquanto as crianças com baixo peso obtiveram uma média de 13,6 $\mathrm{Kg} / \mathrm{m}^{2}$ para os valores de IMC, as com obesidade, uma média de $20,98 \mathrm{Kg} / \mathrm{m}^{2}$ e as crianças classificadas com sobrepeso, minoria, obteve uma média $17.0 \mathrm{Kg} / \mathrm{m}^{2}$.

Na Tabela 3, encontra-se a descrição das características dos participantes segundo a classificação da GMFCS. Observa-se um maior número de crianças classificadas como grau I, com média de IMC de $17,0 \mathrm{~kg} / \mathrm{m}^{2}$; enquanto os participantes classificados como grau II ob-

Tabela 1: Classificação do Índice de Massa Corpórea de acordo com o percentil, segundo o Center for Disease Control.

\begin{tabular}{cc}
\hline Classificação & Percentil do IMC \\
\hline Baixo peso & $<5$ \\
Peso Ideal & $5-85$ \\
Sobrepeso & $85-95$ \\
Obesidade & $>95$ \\
\hline
\end{tabular}


Quadro 1: Características dos participantes segundo sexo, idade, classificação de acordo com distribuição topográfica e uso de sonda.

\begin{tabular}{|c|c|c|c|c|}
\hline Participantes & Sexo & Idade & Distribuição Topográfica & uso de sonda \\
\hline P1 & M & $2 \mathrm{a} 8 \mathrm{~m}$ & hemiplegia & $\mathrm{n}$ \\
\hline $\mathrm{P} 2$ & M & $3 a 10 m$ & quadriplegia & $\mathrm{n}$ \\
\hline P3 & M & $5 a$ & diplegia & $\mathrm{n}$ \\
\hline $\mathrm{P} 4$ & M & $3 a 7 m$ & quadriplegia & $\mathrm{n}$ \\
\hline P5 & $\mathrm{F}$ & $7 \mathrm{a} 5 \mathrm{~m}$ & quadriplegia & $\mathrm{n}$ \\
\hline P6 & M & $10 \mathrm{a} 6 \mathrm{~m}$ & diplegia & $\mathrm{n}$ \\
\hline P7 & M & 10a10m & diplegia & $\mathrm{n}$ \\
\hline P8 & M & $5 \mathrm{a} 11 \mathrm{~m}$ & hemiplegia & $\mathrm{n}$ \\
\hline P9 & M & $6 a 10 m$ & diplegia & $\mathrm{n}$ \\
\hline P10 & $\mathrm{F}$ & $10 \mathrm{a} 4 \mathrm{~m}$ & hemiplegia & $\mathrm{n}$ \\
\hline P11 & M & $8 \mathrm{a} 5 \mathrm{~m}$ & quadriplegia & $\mathrm{n}$ \\
\hline $\mathrm{P} 12$ & $\mathrm{~F}$ & $6 a 11 m$ & quadriplegia & $\mathrm{n}$ \\
\hline $\mathrm{P} 13$ & M & $3 a 3 m$ & diplegia & $\mathrm{n}$ \\
\hline P14 & F & $11 \mathrm{a} 3 \mathrm{~m}$ & hemiplegia & $\mathrm{n}$ \\
\hline P15 & F & $10 \mathrm{a} 4 \mathrm{~m}$ & quadriplegia & $\mathrm{n}$ \\
\hline P16 & M & 12a6m & diplegia & $\mathrm{n}$ \\
\hline P17 & F & $14 a$ & diplegia & $\mathrm{n}$ \\
\hline P18 & M & $9 a$ & hemiplegia & $\mathrm{n}$ \\
\hline P19 & M & $5 \mathrm{a} 10 \mathrm{~m}$ & diplegia & $\mathrm{n}$ \\
\hline P20 & $\mathrm{F}$ & 7 a $5 m$ & hemiplegia & $\mathrm{n}$ \\
\hline
\end{tabular}

tiveram média de IMC de 19,3 kg/m²; os classificados como grau IV obtiveram médias menores em todas as variáveis, inclusive IMC que teve média de $14,1 \mathrm{~kg} / \mathrm{m}^{2}$; e por fim, os indivíduos classificados como grau $\mathrm{V}$ obtiveram uma média de IMC de $18,0 \mathrm{~kg} / \mathrm{m}^{2}$. Nenhuma das crianças participantes do estudo foi classificada como grau III, segundo a GMFCS.

Na Tabela 4, nota-se que houve uma maior frequência de participantes do sexo mas- culino. No entanto, verifica-se que as meninas têm uma média de idade, peso, estatura e IMC maior que a dos meninos.

Na Tabela 5, observam-se os resultados da análise comparativa dos participantes do estudo, segundo o sexo para as variáveis idade, peso, estatura e IMC. Os dados indicam diferença significante em relação à idade, ao peso e à estatura. Não houve diferença significativa para a variável IMC $(\mathrm{p}=0.141)$. 
Quadro 2: Características dos participantes segundo Índice de Massa Corpórea, classificação do Índice de Massa Corpórea e classificação da Escala de classificação da função motora grossa

\begin{tabular}{ccccc}
\hline Participantes & IMC $\left.\mathbf{( K g} / \mathbf{m}^{\mathbf{2}}\right)$ & Percentil & Classificação do IMC & GMFCS \\
\hline P1 & 13,9 & 1 & Baixo peso & I \\
P2 & 12,7 & 1 & Baixo peso & IV \\
P3 & 16,1 & 71 & Peso ideal & I \\
P4 & 14,5 & 10 & Peso ideal & IV \\
P5 & 21,9 & 97 & Obesidade & V \\
P6 & 18,8 & 78 & Peso ideal & II \\
P7 & 16,8 & 48 & Peso ideal & I \\
P8 & 15,2 & 47 & Peso ideal & I \\
P9 & 14,2 & 11 & Peso ideal & I \\
P10 & 14,4 & 7 & Peso ideal & I \\
P11 & 21,2 & 96 & Obesidade & V \\
P12 & 14,8 & 35 & Peso ideal & V \\
P13 & 16,5 & 72 & Peso ideal & I \\
P14 & 22,5 & 91 & Sobrepeso & I \\
P15 & 14,2 & 4 & Baixo peso & V \\
P16 & 15,3 & 61 & Peso ideal & IV \\
P17 & 21,3 & 73 & Peso ideal & I \\
P18 & 16,6 & 60 & Peso ideal & I \\
P19 & 19,8 & 98 & Obesidade & II \\
P20 & 19,8 & 94 & Sobrepeso & I \\
\hline
\end{tabular}

Tabela 2: Frequência encontrada nas variáveis estudadas, idade, peso, estatura e Índice de Massa Corpórea, pela classificação do Índice de Massa Corpórea dos participantes.

\begin{tabular}{clcccc}
\hline \multicolumn{2}{c}{ Classificação do IMC } & Idade $(\boldsymbol{a})$ & Peso $(\mathbf{K g})$ & Estatura $(\boldsymbol{m})$ & $\mathbf{I M C K g / \mathbf { m } ^ { 2 }}$ \\
\hline Baixo peso & $\mathbf{n}$ & 3 & 3 & 3 & 3 \\
& Média & 5,3 & 13,9 & 1,0 & 13,6 \\
& DP & 4,1 & 4,1 & 0,1 & 0,8 \\
& Mínimo & 2,8 & 10,9 & 0,93 & 12,66 \\
& Máximo & 10 & 18,6 & 1,15 & 14,17 \\
\hline Peso Ideal & n & 12 & 12 & 12 & 12 \\
& Média & 7,3 & 24,5 & 1,2 & 16,2 \\
& DP & 3,5 & 10,4 & 0,2 & 2,1 \\
& Mínimo & 2,11 & 11,2 & 0,88 & 14,2 \\
& Máximo & 14 & 48,3 & 1,5 & 21,29 \\
\hline Sobrepeso & n & 2 & 2 & 2 & 2 \\
& Média & 7,0 & 24,0 & 1,2 & 17,0 \\
& DP & 3,2 & 10,4 & 0,2 & 3,1 \\
& Mínimo & 2,7 & 10,9 & 0,9 & 12,7 \\
& Máximo & 14,0 & 48,3 & 1,5 & 22,5 \\
Obesidade & n & 3 & 3 & 3 & 3 \\
& Média & 7 & 26 & 1,1 & 20,98 \\
& DP & 1 & 9,4 & 0,2 & 1,1 \\
& Mínimo & 6 & 15,6 & 0,89 & 19,8 \\
& Máximo & 8 & 34 & 1,25 & 21,92 \\
\hline
\end{tabular}


Tabela 3: Frequência encontrada nas variáveis estudadas, idade, peso, estatura e IMC, pela classificação do GMFCS dos participantes.

\begin{tabular}{|c|c|c|c|c|c|}
\hline \multicolumn{2}{|c|}{ GMFCS } & Idade (a) & Peso $(\mathrm{Kg})$ & Estatura (m) & $I M C \mathrm{Kg} / \mathrm{m}^{2}$ \\
\hline \multirow[t]{5}{*}{$\bar{I}$} & $\mathrm{n}$ & 11 & 11 & 11 & 11 \\
\hline & Média & 7,5 & 26,2 & 1,2 & 17,0 \\
\hline & DP & 3,6 & 11,0 & 0,2 & 2,9 \\
\hline & Mínimo & 2,7 & 12,3 & 0,9 & 13,9 \\
\hline & Máximo & 14,0 & 48,3 & 1,5 & 22,5 \\
\hline \multirow[t]{5}{*}{$\overline{\text { II }}$} & $\mathrm{n}$ & 2 & 2 & 2 & 2 \\
\hline & Média & 8,0 & 25,0 & 1,1 & 19,3 \\
\hline & DP & 2,8 & 13,3 & 0,3 & 0,7 \\
\hline & Mínimo & 6,0 & 15,6 & 0,9 & 18,8 \\
\hline & Máximo & 10,0 & 34,4 & 1,4 & 19,8 \\
\hline \multirow[t]{5}{*}{$\overline{\mathrm{IV}}$} & $\mathrm{n}$ & 3 & 3 & 3 & 3 \\
\hline & Média & 3,1 & 11,5 & 0,9 & 14,1 \\
\hline & DP & 0,3 & 0,9 & 0,0 & 1,4 \\
\hline & Mínimo & 2,9 & 10,9 & 0,9 & 12,7 \\
\hline & Máximo & 3,5 & 12,5 & 0,9 & 15,3 \\
\hline \multirow[t]{5}{*}{$\bar{V}$} & $\mathrm{n}$ & 4 & 4 & 4 & 4 \\
\hline & Média & 7,8 & 26,7 & 1,2 & 18,0 \\
\hline & DP & 1,7 & 6,4 & 0,1 & 4,1 \\
\hline & Mínimo & 6,0 & 18,6 & 1,2 & 14,2 \\
\hline & Máximo & 10,0 & 34,0 & 1,3 & 21,9 \\
\hline \multirow{5}{*}{ Total } & $\mathrm{n}$ & 20 & 20 & 20 & 20 \\
\hline & Média & 7,0 & 24,0 & 1,2 & 17,0 \\
\hline & DP & 3,2 & 10,4 & 0,2 & 3,1 \\
\hline & Mínimo & 2,7 & 10,9 & 0,9 & 12,7 \\
\hline & Máximo & 14,0 & 48,3 & 1,5 & 22,5 \\
\hline
\end{tabular}

Tabela 4: Frequência encontrada nas variáveis estudadas, idade, peso, estatura e IMC, por sexo dos participantes.

\begin{tabular}{clcccc}
\hline Sexo & Idade (a) & & Peso $(\mathbf{K g})$ & Estatura $(\boldsymbol{m})$ & $\mathbf{I M C K g} / \mathbf{m}^{\mathbf{2}}$ \\
\hline Feminino & $\mathrm{n}$ & 7 & 7 & 7 & 7 \\
& Média & 9,3 & 30,8 & 1,3 & 18,4 \\
& DP & 2,8 & 11,1 & 0,1 & 3,8 \\
& Mínimo & 6,0 & 18,6 & 1,1 & 14,2 \\
& Máximo & 14,0 & 48,3 & 1,5 & 22,5 \\
\hline Masculino & $\mathrm{n}$ & 13 & 13 & 13 & 13 \\
& Média & 5,7 & 20,3 & 1,1 & 16,3 \\
& DP & 2,7 & 8,2 & 0,2 & 2,5 \\
& Mínimo & 2,7 & 10,9 & 0,9 & 12,7 \\
& Máximo & 10,0 & 34,4 & 1,4 & 21,2 \\
\hline \multirow{2}{*}{ Total } & n & 20 & 20 & 20 & 20 \\
& Média & 7,0 & 24,0 & 1,2 & 17,0 \\
& DP & 3,2 & 10,4 & 0,2 & 3,1 \\
& Mínimo & 2,7 & 10,9 & 0,9 & 12,7 \\
& Máximo & 14,0 & 48,3 & 1,5 & 22,5 \\
\hline
\end{tabular}


Tabela 5: Comparação entre os indivíduos, segundo o sexo, por meio do teste t de Student para grupos independentes, segundo as variáveis numéricas: idade, peso, estatura e IMC.

\begin{tabular}{lccccc}
\hline \multicolumn{1}{c}{ Variável } & Sexo & $\boldsymbol{n}$ & Média & $\boldsymbol{D P}$ & $\boldsymbol{p}$ \\
\hline Idade & Feminino & 7 & 9,3 & 2,8 & 0,013 \\
& Masculino & 13 & 5,7 & 2,7 & \\
\hline Peso & Feminino & 7 & 30,8 & 11,1 & 0,027 \\
& Masculino & 13 & 20,3 & 8,2 & \\
\hline Estatura & Feminino & 7 & 1,3 & 0,1 & 0,037 \\
& Masculino & 13 & 1,1 & 0,2 & \\
\hline IMC & Feminino & 7 & 18,4 & 3,8 & 0,141 \\
& Masculino & 13 & 16,3 & 2,5 & \\
\hline
\end{tabular}

$\mathrm{p} \leq 0,05$

\section{DISCUSSÃO}

Os achados deste estudo corroboram as afirmações de Johnston e Hagberg ${ }^{14}$ e Shankaran ${ }^{15}$ que parece ser mais comum encontrar meninos acometidos de PC do que meninas.

A média do IMC mais alta apresentada pelas meninas pode ser explicada pelos valores das médias de estatura, peso e idades comumente maiores em meninas do que em meninos, uma vez que os participantes do sexo masculino eram mais novos, mais baixos e mais leves. Ainda, deve-se levar em consideração que meninas desenvolvem-se e atingem a maturidade antes dos meninos ${ }^{16}$.

Em contradição aos estudos dos indicadores antropométricos em pacientes com paralisia cerebral, realizados em países europeus, na América do Norte e Ásia - que têm demonstrado prejuízo dos indicadores antropométricos associados à desnutrição ${ }^{7}$ e também ao de Campos et al. ${ }^{6}$ que estudou a influência das competências alimentares em crianças com PC, os resultados obtidos nesta pesquisa não indicaram um elevado índice de desnutrição nas crianças avaliadas, a maio- ria das crianças foi classificada com peso ideal para a idade e sexo.

Linhares ${ }^{4}$ também realizou seu estudo com participantes acometidos de PC atendidos em um centro de referência no atendimento a crianças com necessidades especiais e, assim como no presente trabalho, os resultados obtidos demonstraram um predomínio de crianças com peso ideal. Isso pode ser justificado devido ao acompanhamento multidisciplinar de fisioterapia, terapia ocupacional e fonoaudiologia disponível nos centros de reabilitação e também ao acompanhamento dessas crianças, que apresentam alterações na deglutição de alimentos, por nutricionistas.

Outro item que deve ser considerado, nesta pesquisa, é o acompanhamento daqueles com alterações na deglutição, no ambulatório de disfagia disponível no centro de reabilitação frequentado pelos participantes. Além disso, nenhum dos participantes fazia uso de qualquer tipo de sonda para alimentar-se, o que reflete um comprometimento menor na função digestiva.

Esse acompanhamento multidisciplinar disponível no centro de reabilitação ao qual as crianças pertenciam, também justifica a maioria dos participantes ter sido classificada como grau I na escala GMFCS; enfatizando a impor- 
tância do atendimento fisioterapêutico, fonoaudiológico e da terapia ocupacional para a minimização das incapacidades e comprometimentos motores desses indivíduos.

Também foi possível observar que os indivíduos classificados com baixo peso apresentaram uma média de idade menor do que os demais, o que pode sugerir que pais de crianças mais novas ainda não tenham se atentado para esse fator clínico, ou não tenham informações suficientes para tratar da situação alimentar.

De acordo com Hurvitz et al. ${ }^{8}$, os indivíduos classificados com sobrepeso e obesidade apresentam um comportamento sedentário. Estudos do mesmo autor mostram que indivíduos com PC são menos ativos que os indivíduos sem alterações e que, quando se submetem à atividade física, apresentam limitações na acessibilidade de treinamentos.

Além disso, o mesmo autor sugere que o IMC elevado, acima do ideal, está diretamente associado a fatores de risco como colesterol elevado e diabetes.

Tais informações refletem a importância da equipe multiprofissional, principalmente dos fisioterapeutas, em se atentarem para os valores de IMC, adaptando atividades físicas e buscando novos meios para um controle do sobrepeso e obesidade, tentando minimizar esses índices elevados, transformando-os em valores ideais.

Linhares $^{4}$ afirmou que a gravidade do tipo de PC está diretamente relacionada à dificuldade de deglutição, ao aumento de sintomas de disfagia e também a maiores complicações do estado nutricional. Assim, o grau de comprometimento motor, talvez, possa ser considerado um fator preditor para alterações nu- tricionais. Embora os resultados obtidos, nesta pesquisa, não tenham demonstrado relação total e direta entre a classificação GMFCS e classificação do IMC, alguns indivíduos (8/20 - 40\%) foram classificados como grau I na GMFCS e com peso ideal, indicando que o menor comprometimento motor possa ser fator preditor para alterações alimentares.

Portanto, não houve relação entre os valores de IMC e a classificação da GMFCS, uma vez que os indivíduos gravemente acometidos, correspondentes ao grau V na GMFCS, foram classificados como baixo peso, obesidade e peso ideal; e aqueles identificados como grau I na GMFCS, ou seja, com pouco comprometimento na função motora grossa, foram classificados como baixo peso, sobrepeso e peso ideal.

O número reduzido de participantes; a escala da GMFCS, a qual se baseia em habilidades de sentar, manter-se em pé e andar para classificar os participantes e não habilidades diretamente relacionadas à ingesta de alimentos; e também o uso do IMC para avaliar o estado nutricional dos indivíduos, o qual não diferencia massa magra de massa gorda, podem justificar essa falta de relação entre o IMC e classificação da GMFCS.

Apesar dessas limitações, este estudo mostrou a importância do acompanhamento multidisciplinar às crianças com paralisia cerebral e também da avaliação da função motora e nutricional.

Principalmente pelo fato de muitas crianças com paralisia cerebral apresentarem alterações na função motora oral e dificuldades para se alimentar, o que pode prejudicar não somente o crescimento dessas, como também influenciar no seu processo de reabilitação. 


\section{REFERÊNCIAS}

1. Mancini MC Alves ACM, Schaper C, Figueiredo EM, Sampaio RF, Coelho ZAC et al. Gravidade da paralisia cerebral e desempenho funcional. Rev. Bras. Fisioter. 2004; 8(3): 253-260

2. Lima CLA, Fonseca LF. Paralisia cerebral: neurologia, ortopedia, reabilitação. Rio de Janeiro: Guanabara Koogan; 2004.

3. Ratliffe KT. Fisioterapia Clínica pediátrica. São Paulo: Santos; 2000.

4. Linhares FMP. Avaliação do estado nutricional de crianças com disfagia por paralisia cerebral. [Dissertação de Mestrado] Santa Maria: Universidade Federal de Santa Maria; 2004.

5. Neto FT. Nutrição Clínica. Rio de Janeiro: Guanabara Koogan; 2003.

6. Campos MA, Kent-Smith L, Santos CC. Efeito das competências alimentares no estado nutricional: de crianças portadoras de paralisia cerebral. Acta Med Port. 2007; 20: 21-27.

7. Caram ALA. Avaliação nutricional antropométrica de crianças com paralisia cerebral. [Dissertação de mestrado] Campinas: Faculdade de Ciências Médicas da Universidade Estadual de Campinas; 2006.

8. Hurvitz EA, Green LB, Hornyak JE, Khurana SR, Koch LG. Body mass index measures in children with cerebral palsy related to gross motor function classification: a clinic-based study. Am J Phys Med Rehabil. 2008; 87: 395 - 493.
9. Stevenson, R.D. Use of segmental measures to estimate stature in children with cerebral palsy. Arch Pediatr Adolesc Med.1995;149(6):658-662.

10. Palisano, R.; Rosenbaum, P.; Bartlett, D., Livingston, M. Content validity of the expanded and revised Gross Motor Function Classification System. Dev Med Child Neurol. 2008;50(10):744-50.

11. Palisano, R; Rosenbaum, P; Walter, S; Russell, D; Wood, E; galuppi, B. Development and reliability of a system to classify gross motor function in children with cerebral palsy. Dev Med Child Neurol, 1997; 39(4): 214-23.

12. Feltrin C, Morais MB, Speridião PGL, Neto UF. Índices antropométricos mais utilizados na avaliação do estado nutricional na infância. The Electronic Journal of Pediatric Gastroenterology, Nutrition and Liver Diseases. [periódico online] 2004; [Acesso em 20 de jan. 2009] 8(4) Disponível em: $<$ http://www.e-gastroped.com.br/mar05/ indicesantrop.htm>.

13. Armitage P, Berry G. Estatística para la investigación biomédica. $3^{\text {a }}$. Ed. Madrid: Harcourt Brace, 1997.

14. Johnston MV, Hagberg H. Sex and the pathogenesis of cerebral palsy. Dev Med Child Neurol, 2007; 49:74-78.

15. Shankaran S. Prevention, diagnosis, and treatment of cerebral palsy in near-term and term infants. Clin Obstet Gynecol. 2008; 51(4): 829-839.

16. Duarte MFS. Maturação física: uma revisão da literatura, com especial atenção à criança brasileira. Cad. Saúde Pub. 1993; 9 (supl.1): 71-84.

Recebido em: 06/set./10

Modificado em: 02/dez./10

Aceito em: 28/dez./10 